NIKHEF-H 94-17

INLO-PUB-4/94

\title{
Weight optimization in multichannel Monte Carlo ${ }^{\prime}$ Ronald Kleiss?
}

\author{
NIKHEF-H, Amsterdam, the Netherlands \\ Roberto Pittau? \\ Instituut-Lorentz, University of Leiden, the Netherlands
}

\begin{abstract}
We discuss the improvement in the accuracy of a Monte Carlo integration that can be obtained by optimization of the a-priori weights of the various channels. These channels may be either the strata in a stratified-sampling approach, or the several 'approximate' distributions such as are used in event generators for particle phenomenology. The optimization algorithm does not require any initialization, and each Monte Carlo integration point can be used in the evaluation of the integral. We describe our experience with this method in a realistic problem, where an effective increase in program speed by almost an order of magnitude is observed.
\end{abstract}

\footnotetext{
${ }^{1}$ This research has been partly supported by EU under contract number CHRX-CT-0004.

${ }^{2}$ e-mail: t30@nikhefh.nikhef.nl

${ }^{3}$ e-mail: pittau@rulgm0.leidenuniv.nl
} 
In almost all Monte Carlo integrations, an effort must be made to reduce the variance of the integrand [1, 2]. One of the currently popular approaches of variance reduction is the so-called stratified sampling technique, where the integration region is divided in a number of bins, with a (usually) predetermined number of integration points in each bin. An example of this technique is the program VEGAS [4], in which the bins are automatically redefined from time to time so as to reduce the integration error. Another approach is that of importance sampling, where various techniques are used to obtain (pseudo-)random variables that have a non-uniform rather than a uniform distribution: one tries to generate a density of Monte Carlo points that is larger in those parts of the integration region where also the integrand is large, thus reducing the error (note that this will work only when the integrand has large positive values; large negative values do not lend themselves to probabilistic modelling). Importance sampling is widely used in event generators for particle phenomenology: also, the Metropolis algorithm [5] used in statistical physics is actually a form of importance sampling.

In the construction of event generators for particle phenomenology, the aim is usually to generate Monte Carlo points in some phase space of final-state momenta (and spins), with a density proportional to a predetermined multidifferential cross section. Often, such a cross section exhibits, in different regions of phase space, peaks that find their best description in terms of different sets of phase space variables. An example is provided by bremsstrahlung in a particle collision process, where the bremsstrahlung quanta are emitted, in the different Feynman diagrams, by different particles. It is customary, in such a case, to generate each peaking structure with a different mapping of (pseudo-)random numbers: the particular mapping used to generate an event is then chosen randomly, using a predtermined set of probabilities, which we shall call a-priori weights. It is the aim of this paper to indicate how these a-priori weights lend themselves to optimization.

First, we establish some notation. The function to be integrated is $f(\vec{x})$, where $\vec{x}$ denotes a set of phase-space variables. Each distinct mapping of random numbers into $\vec{x}$ is called a channel, and each channel gives rise to a different (non-uniform) probability density, that we denote by $g_{i}(\vec{x}), i=1,2, \ldots, n ; n$ is the number of channels in our multichannel Monte Carlo. Each density $g_{i}$ is of course nonegative and normalized to unity: $\int g_{i}(\vec{x}) d \vec{x}=1$. The a-priori weights are denoted by $\alpha_{i}$, and also these must be a partition of unity: $\alpha_{i} \geq 0$ and $\sum_{i=1}^{n} \alpha_{i}=1$. If the channels are picked at random, with probability $\alpha_{i}$ for channel $i$, the total probability density of the obtained sample of $\vec{x}$ values is $g(\vec{x})=\sum_{i=1}^{n} \alpha_{i} g_{i}(\vec{x})$, which is also nonegative and normalized to unity. Note that we may take the $g_{i}(\vec{x})$ to be linearly independent. The weight assigned to each Monte Carlo point must, then, be $w(\vec{x})=f(\vec{x}) / g(\vec{x})$. The expectation value of the result of this Monte Carlo integration, and its variance, 
then follow from

$$
\begin{gathered}
I=\langle w(\vec{x})\rangle=\int d \vec{x} g(\vec{x}) w(\vec{x})=\int d \vec{x} f(\vec{x}) \\
W(\alpha)=\left\langle w(\vec{x})^{2}\right\rangle=\int d \vec{x} g(\vec{x}) w(\vec{x})^{2}=\int d \vec{x} \frac{f(\vec{x})^{2}}{g(\vec{x})} .
\end{gathered}
$$

Here we have indicated the dependence of $W$ on the set $\alpha_{i}$. The expected error of the integration, for $N$ points, is $\left[\left(W(\alpha)-I^{2}\right) / N\right]^{1 / 2}$. It is the quantity $W(\alpha)$ that we may try to minimize by adjustment of the $\alpha_{i}$. Note that, since $I$ does not depend on $g(\vec{x})$, we may change the $\alpha_{i}$ during the integration, even from one Monte Carlo point to the next. Therefore, any such optimization procedure will always lead to an unbiased result.

The extremum of $W(\alpha)$ (homogeneous of degree -1 in the $\alpha_{i}$ ) on the simplex described by the $\alpha_{i}$ is obtained for those values $\bar{\alpha}_{i}$ for which $W_{i}(\bar{\alpha})=W(\bar{\alpha})$ for all $i$, where

$$
W_{i}(\alpha) \equiv-\frac{\partial}{\partial \alpha_{i}} W(\alpha)=\int d \vec{x} g_{i}(\vec{x}) w(\vec{x})^{2}
$$

That this extremum is, indeed, a minimum can be proven simply. For let $\alpha_{i}=\bar{\alpha}_{i}+\beta_{i}$, where $\beta_{i}$ is small. Then, $\sum_{i=1}^{n} \beta_{i}=0$, and we have

$$
W(\alpha)=W(\bar{\alpha})+\frac{1}{2} \int d \vec{x} \frac{f(\vec{x})^{2}}{g(\vec{x})^{3}}\left(\sum_{i=1}^{n} \beta_{i} g_{i}(\vec{x})\right)^{2}+\mathcal{O}\left(\beta_{i}^{3}\right) .
$$

In general, we cannot prove that the minimum is unique. It is interesting to study some simple cases. In the first place, suppose that $f(\vec{x})$ is dependent with the set $g_{i}(\vec{x})$, that is, there are constants $\gamma_{i} \geq 0$ such that $f(\vec{x})=\sum_{i=1}^{n} \gamma_{i} g_{i}(\vec{x})$. We then have $\int d \vec{x} f(\vec{x})=\sum_{i=1}^{n} \gamma_{i}$, and the minimum for $W(\alpha)$ is reached at $\bar{\alpha}_{k}=\gamma_{k} / \sum_{i=1}^{n} \gamma_{i}$, in which case $W_{k}(\alpha)=W(\alpha)=\left(\sum_{i=1}^{n} \gamma_{i}\right)^{2}$. Of course, in this case the Monte Carlo error is zero.

A second case of interest is that of stratified sampling. This is described, in our formalism, by a set of channels $g_{i}(\vec{x})$ that each restrict the values $\vec{x}$ to a piece of phase space, a bin. The bins must be disjoint and together make up the whole phase space volume. That is,

$$
g_{i}(\vec{x})=\frac{1}{\omega_{i}} \theta_{i}(\vec{x})
$$

where $\theta_{i}(\vec{x})$ is the characteristic function of bin $i$, and $\omega_{i}$ its volume, $\omega_{i}=\int d \vec{x} \theta_{i}(\vec{x})$. In that case,

$$
W_{i}(\alpha)=\frac{\omega_{i}}{\alpha_{i}^{2}} \int d \vec{x} \theta_{i}(\vec{x}) f(\vec{x})^{2}
$$

and we recover the well-known result [2] that the error is minimized if each bin contributes the same amount to the total variance. An illustrative limiting case is that where all the bins are vanishingly small and have equal volume $\omega$, so that 
$\int d \vec{x} f(\vec{x}) \theta_{i}(\vec{x})=\omega f\left(\vec{x}_{i}\right)$, where $\vec{x}_{i}$ is the center of each bin. We then have, again, that the optimal case is $g(\vec{x}) \propto f(\vec{x})$.

A remark is in order here. Stratified sampling, as usually defined, uses a deterministic, rather than a random, choice of the channels. Typically, one first generates points in bin 1, then in bin 2, and so on. In our formalism, this is covered by going over, for the variable that determines the choice of channel, from a pseudorandom variable to a strictly uniform one. Not surprisingly, one then has to predetermine the exact number of Monte Carlo points, since a uniform point set can only be generated in a deterministic way if the total number of points is known. Whether this is an attractive or a repulsive feature of our formalism, is largely a matter of taste.

The following procedure suggests itself. Start the Monte Carlo, using some set $\alpha_{i}$, picked either randomly or on the basis of some information on the behaviour of $f(\vec{x})$ and $g(\vec{x})$. After generating a number of Monte Carlo points (a few hundred, say) in each channel, estimate the $W_{i}(\alpha)$, using

$$
W_{i}(\alpha)=\left\langle\frac{g_{i}(\vec{x})}{g(\vec{x})} w(\vec{x})^{2}\right\rangle .
$$

Then, use this information to find an improved set of $\alpha_{i}$. Repeat this procedure until no further improvement is found; the results of all iterations may be added in the final integral estimate. Obviously, when some $W_{i}$ is large (small), the optimization should give a new $\alpha_{i}$ that is larger (smaller) than the old one. The above examples naturally suggest an optimization prescription. In the case of stratified sampling, the optimum was reached when $W_{i}(\alpha)=c \alpha_{i}^{-2}$, with some constant $c$ independent of $i$. This implies that, supposing the idealized case where each $W_{i}(\alpha)$ is estimated with zero error (this does not mean that the integral has zero error!), the choice of new $\alpha_{i}$ according to

$$
\alpha_{i}^{\text {new }} \propto \alpha_{i} \sqrt{W_{i}(\alpha)},
$$

will give immediately the optimal set $\bar{\alpha}_{i}$, irrespective of the choice of the initial set $\alpha_{i}$. Therefore, we propose to use this optimization prescription. It should be observed that the extra computational burden is actually quite small, since, whichever the actual channel is picked to generate an $\vec{x}$, one always has to compute the contributions from all channels in the calculation of $g(\vec{x})$.

There are a few points to be noted here. In the first place, the new $\alpha_{i}$ have to be renormalized to as to sum to unity: hence, it is irrelevant whether we use $W_{i}$, or $W_{i} / W$, or $W_{i} / \sum_{k=1}^{n} W_{k}$, or any other scaled version. Secondly, in practice the values of the $W_{i}$ will have their own Monte Carlo error, and the convergence will be less than immediate, even for stratified sampling. This may actually be considered an advantage, since numerical methods with slower convergence tend, in many cases, to be the more robust ones. Thirdly, for non-stratified sampling, the $\alpha_{i}$ will influence each other, and our prescription cannot be shown to be the best possible. However, 
in practice, the different $g_{i}(\vec{x})$ usually put their largest mass in quite different regions of phase space. Channels that overlap to a large extent will show a lot of 'cross-talk', but then again, the way they split their a-priori weights between them is less important for the overall error (note that if a set of channels is linearly dependent, only the sum of their a-priori weights will be determined).

As in all numerical optimization schemes, many alternatives and improvements of approach can be envisaged. We mention only two. In the first place, one may decide to use, in the determination of $W_{i}$, only the points actually generated with the corresponding channel $g_{i}(\vec{x})$, or using the points from all channels. The last choice, which we prefer, and to which Eq.(6) corresponds, has the advantage that the error on $W_{i}$ will be smaller, and the drawback that then these errors will be correlated. In the second place, nothing forces one to actually use a putative updated set of a-priori weights: on the basis of a given sample of Monte Carlo points $\vec{x}$, together with the values of $f(\vec{x})$ and the $g_{i}(\vec{x})$, one is free to study the behaviour of $W(\alpha)$ for any set $\alpha_{i}$. This might be convenient if one wants to choose between various alternative optimization prescriptions before actually going to the next iteration. Of course, if the envisaged new set of 'virtual' $\alpha_{i}$ differs too dramatically from the a-priori weights that were in fact used to obtain the point sample, the error in the 'virtual' $W_{i}(\alpha)$ will be large. Since the method outlined in this paper is quite new, we have not yet studied such embellishments.

We shall now discuss the application of the proposed method in practice, first in a very simple example of stratified-sampling Monte Carlo, and secondly in an actual event generator.

We start in one dimension, by integrating the function $f(x)=e^{-x}$ from 0 to $\infty$. We use three channels, given by

$$
\begin{aligned}
& g_{1}(x)=\theta(x) \theta(1-x), \\
& g_{2}(x)=\theta(x-1) \theta(2-x), \\
& g_{3}(x)=\theta(x-2) /(x-1)^{2},
\end{aligned}
$$

in other words, the two uniform distributions between 0 and 1 and between 1 and 2 , respectively, and a 'tail' up from 2 . The a-priori weights are set to $\alpha_{1}=\alpha_{2}=\alpha_{3}=1 / 3$ at the beginning. We generate 100,000 events to integrate $f(x)$, employing three different strategies. In the first case, we integrate without optimization. In the second case, we optimize once, after the first 1,000 points. In the third case, we optimize 9 times, after each set of 2,000 points. We monitor the estimated Monte Carlo error on the integral at every hundredth point. The results are given in the figure. The case where we do not optimize shows the classical $1 / \sqrt{N}$ behaviour (a straight line with slope $-1 / 2$ in the double-logarithmic plot). The effect of optimization in the two other cases is clear: immediately after optimization, the error decreases faster 


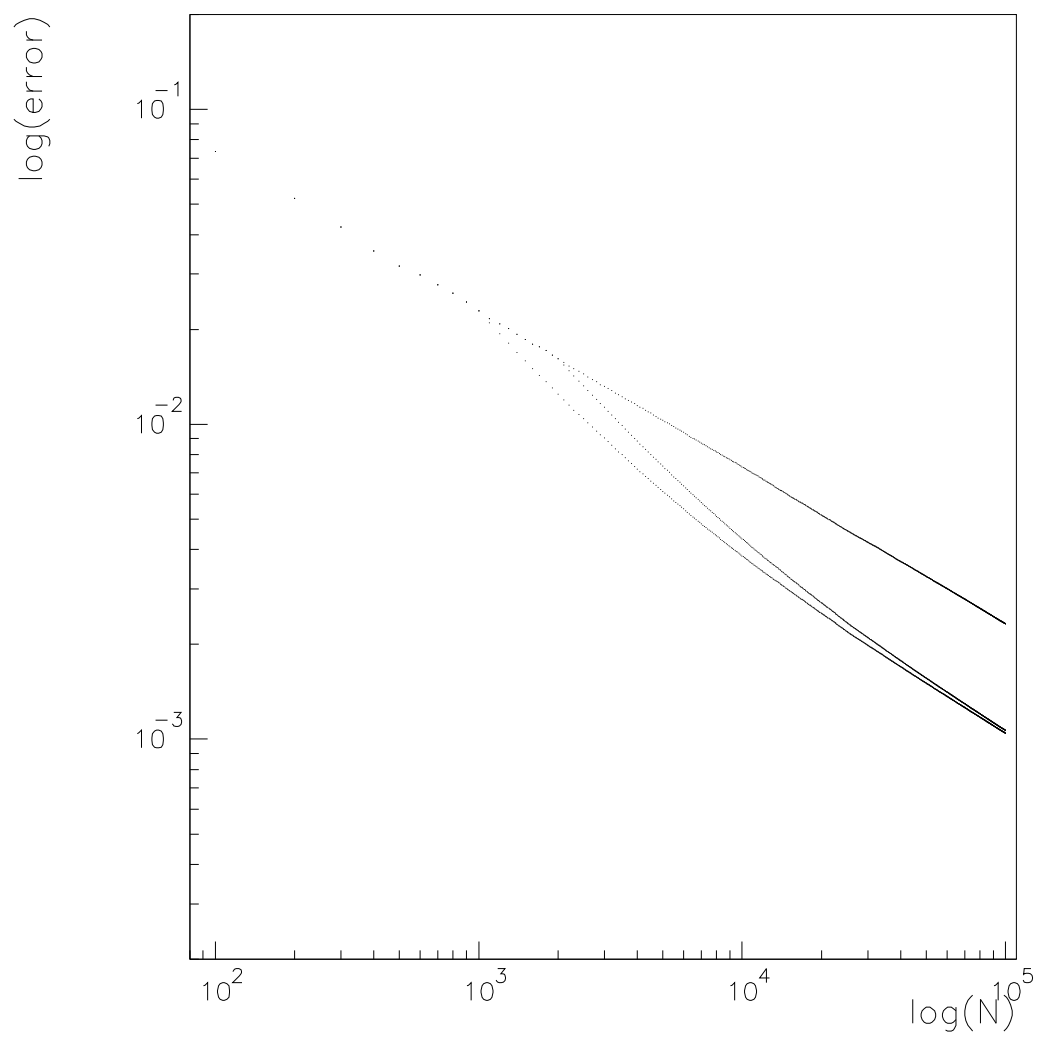

Figure 1: Behaviour of the Monte Carlo error under various optimizations.

than before. Asymptotically, its behaviour is seen to settle again as $1 / \sqrt{N}$, but with a smaller proportionality factor: the variance has been reduced. The non-linear behaviour just after optimization is due to the mixture of sets of Monte Carlo points with different variance. The cases of a single and of multiple optimization steps are seen to be asymptotically equivalent: both optimizations have brought us close to the theoretical optimum. In fact, for this simple case we can in fact compute $\bar{\alpha}_{1}=0.626$, $\bar{\alpha}_{2}=0.230$, and $\bar{\alpha}_{3}=0.144$, whereas single optimization gave $\alpha_{1}=0.623, \alpha_{2}=0.229$ and $\alpha_{3}=0.147$, and multiple optimization $\alpha_{1}=0.636, \alpha_{2}=0.223$, and $\alpha_{3}=0.140$. In this case, the simpler approach actually happens to be a bit closer to the theoretical optimum! Since we are dealing here with stratified sampling, where the approach to the optimum can be very fast ( $c f$. the discussion above) this is not surprising.

In the above example, the error was reduced by about a factor of two (equivalent to an increase by a factor 4 in the statistics). This moderate improvement follows 
from the fact that our starting values for the a-priori weights happened not to be very far from the optimum set. We may expect, therefore, that the improvement in the error may be larger when the $\bar{\alpha}_{i}$ are very dissimilar; and this is actually borne out by our second example, as we shall see.

For every numerical method, simple examples can always be found where it works very well - its real merit can only be judged in a problem of actual interest, such as the following one. We have applied our optimization approach to the real-life calculation of a complicated cross section, namely that of electron-positron annihilation, at LEP200 energies, into an electron-positron pair and an (electron)neutrinoantineutrino pair. Processes such as this one are characterized by a large number of contributing Feynman diagrams, exhibiting strong peaks in many different regions of phase space, and the presence of complicated kinematical cuts which render an analytic treatment impossible. Our Monte Carlo studies of this and similar processes are reported on in [3], but here we only discuss the optimization of the a-priori weights that we used. For this process the number of channels $g_{i}(\vec{x})$ is 39 , that initially all have the same a-priori weight (other four-fermion processes are described by different numbers of channels, depending on the kinematics and the appropriate Feynman diagrams). Optimization is applied after a fixed number of points have been generated, where the steps between successive optimizations is slowly increased (this is based on the expected and observed fact that, after an optimization step, the next improvement needs more statistics to be effective). At the end of each step, we compute a measure of the disrepancy between the values of the 39 different $W_{i}(\alpha)$ :

$$
D=\max _{i, j}\left|W_{i}-W_{j}\right|
$$

Hence, $D$ measures how well the set of $\alpha_{i}$ approximates the behaviour of the optimal set. At the end of (in this case) 7 steps, we then determine which of the 7 sets $\alpha_{i}$ performed the best in terms of $D$ : usually, this is one of the last sets obtained. This set is then used in the rest of the simulation, with a high-statistics run. In this way, we feel that we strike a balance between the benefits of optimization and the possibility that the last set of $\alpha_{i}$ obtained is actually a bit worse than a previous one. The actual numbers come out as follows, for 100,000 Monte Carlo points. Wihtout optimization of the a-priori weights, the integral (a cross section $\sigma$ of 0.17360 picobarns) has an estimated error $\delta$ of 0.00304 picobarn, which compares well (that is, to within an order of magnitude) with the rule of thumb that $\delta \sim \sigma / \sqrt{N}$ : it means that our choice of channels is reasonably good at describing the various peaks in the cross section. Now, we turn to the case with optimization. The 7 successive values of $D$ are given in the table, together with the value of $N$ at which they were measured. 


\begin{tabular}{|c|c|c|}
\hline \hline iter. & $N$ & $D$ \\
\hline 1 & 5,000 & 2.804 \\
2 & 10,000 & 0.416 \\
3 & 15,000 & 0.295 \\
4 & 20,000 & 0.277 \\
5 & 30,000 & 0.254 \\
6 & 40,000 & 0.349 \\
7 & 50,000 & 0.241 \\
\hline \hline
\end{tabular}

It is seen that $D$ tends to decrease, as expected, except for iteration 6 : in our case we use the set $\alpha_{i}$ for iteration 7 ; had we employed only 6 iterations, we would have chosen the set for iteration 5. The final part of the integration consists, then, with a run of 50,000 points, using the set of $\alpha_{i}$ obtained in iteration 7 . With this optimization, the integral comes out as 0.17066 picobarn with an arror of 0.00113 picobarn: a reduction of nearly a factor of 3 . Note that, since the optimization takes up, a non-negligible fraction of the Monte Carlo points, the asymptotic improvement is actually somewhat larger (we are not in the asymptotic regime that can be observed in the figure): the error would, without optimization, be obtained only for ten times as many Monte Carlo points. The $\bar{\alpha}_{i}$ are actually far from uniform: we started with $\alpha_{i} \sim 0.025$ for all $i$, and optimization leads to values ranging from 0.63 down to 0.0000066 , a span of 5 orders of magnitude! This difference between the initial and final set of a-priori weights explains the good performance of optimization. Incidentally, note that, in the Monte Carlo program, points sometimes have to be assigned zero weight (for instance, if they fall outside the experimentally defined cuts): without optimization, we have 40,848 such events out of 100,000: with optimization, there are 29,111 such points left - another indication of self-adjustement at work.

In conclusion, we have described a simple method for automatically improving the performance of multichannel Monte Carlo, that is, any Monte Carlo integration where a decision is made at some point, using predetermined probabilities, on where, or how, to choose the next point (stratified and importance sampling, respectively). The strategy is very modest in terms of time or memory requirements, and has been seen to perform well in a realistic application to a very nontrivial physical problem. We feel that its use deserves consideration in complicated event-generating simulation programs such as are used in high-energy physics.

\section{References}

[1] J.M. Hammersley and D.C. Handscomb, Monte Carlo Methods, (London, Methuen, 1964).

[2] F. James, Rep. Prog. Phys. 43 (1980) 1145. 
[3] F.A. Berends, R. Kleiss and R. Pittau, All electroweak four-fermion processes in electron-positron collisions, Leiden/NIKHEF preprint INLO-PUB1/94, NIKHEF-H/94-08 (1994) (to be published in Nucl. Phys. B).

[4] G. Peter Lepage, J. Comp. Phys. 27 (1978) 192, and Cornell preprint CLNS80/447 (1980).

[5] G. Bhanot, Rep. Prog. Phys. 51 (1988) 429. 